\title{
Effects of Oxime K203 and Oxidative Stress in Plasma of Tabun Poisoned Rats ${ }^{\dagger}$
}

\author{
Suzana Berend, ${ }^{\mathrm{a}, *}$ Ana Lucić Vrdoljak, ${ }^{\mathrm{a}}$ Kamil Musilek, ${ }^{\mathrm{b}, \mathrm{c}}$ Kamil Kuča, ${ }^{\mathrm{b}, \mathrm{cd}}$ and Božica Radić ${ }^{\mathrm{a}}$ \\ ${ }^{a}$ Institute for Medical Research and Occupational Health, Ksaverska c. 2, P.O. Box 291, 10000 Zagreb, Croatia \\ ${ }^{\mathrm{b}}$ Department of Toxicology, Faculty of Military Health Sciences, University of Defense, \\ Třebešská 1575, Hradec Králové, Czech Republic \\ ${ }^{\mathrm{c}}$ Department of Chemistry, Faculty of Sciences, J.E. Purkinje University, Usti nad Labem, Czech Republic \\ ${ }^{\mathrm{d}}$ Center of Advanced Studies, Faculty of Military Health Sciences, University of Defense, \\ Třebešská 1575, Hradec Králové, Czech Republic
}

RECEIVED DECEMBER 9, 2010; REVISED NOVEMBER 16, 2011; ACCEPTED MAY 8, 2012

\begin{abstract}
The highly toxic nature of tabun has been known for many years, but there are still serious limitations to antidotal therapy. In this study, we used rats as an experimental model to evaluate the efficiency of bispyridinium para-oxime K203 as therapy against tabun poisoning as well as to examine if induction of oxidative stress is linked to organophosphate toxicity. K203 showed high potency in counteracting tabun poisoning. Either alone or in combination with atropine, this oxime significantly increased cholinesterase activity at 0.5 and $1 \mathrm{~h}$ compared to untreated rats poisoned with tabun. Simultaneous measurements of markers of oxidative stress (lipid peroxidation and superoxide dismutase) showed that tabun poisoning, but also therapy (oxime alone or oxime plus atropine) applied immediately after tabun poisoning, could generate free radical species that may cause oxidative stress in rats. (doi: 10.5562/cca1811)
\end{abstract}

Keywords: tabun, therapy, K203, cholinesterase, lipid peroxidation, superoxide dismutase

\section{INTRODUCTION}

Organophosphorus (OP) compounds such as nerve agents (tabun, soman, sarin and VX) and pesticides [paraoxon, chlorpyrifos, and tetraethyl pyrophosphate (TEPP)] constitute an extremely toxic group of compounds. ${ }^{1}$ Nerve agents like tabun are still a potential threat for both military and civilian populations. The toxic symptomatology of OP is caused primarily by irreversible inhibition of cholinesterases, in particular acetylcholinesterase (AChE). Inhibition by OP compounds is manifested through conjugation of a phosphorous group from OP to the catalytic serine residue at the active site of the enzyme. ${ }^{2} \mathrm{AChE}$ is a vitally important enzyme that hydrolyzes the neurotransmitter acetylcholine $(\mathrm{ACh})$, which is involved in the numerous cholinergic pathways in both the central and peripheral nervous system. Consequently, inhibition of AChE results in severe cholinergic toxic signs caused by increased concentration of ACh at cholinergic nerve-nerve and nervemuscle synapses. ${ }^{3}$ A combination of anticholinergics (atropine) and AChE reactivators (oximes) is currently used for the treatment of OP intoxications. ${ }^{4}$ Atropine blocks muscarinic acetylcholine-receptors and prevents them from further stimulation by ACh. In this way, atropine relieves the symptoms of OP poisoning, but it does not threat the poisoning itself. $\mathrm{AChE}$ reactivators are mono- or bis-quaternary pyridinium salts bearing in their molecule a functional oxime group able to reactivate inhibited AChE by displacing the phosphoryl residue from the active site and restoring the enzyme activity. Pralidoxime (2-PAM), TMB-4, obidoxime and HI-6 are the most commonly used oximes for the treatment of OP exposure. However, reactivation by these oximes is not possible in all cases of OP poisoning, especially in the case of the nerve agent tabun. Namely, tabunphosphorylated $\mathrm{AChE}$ is resistant to reactivation due to low electrophilicity of the phosphoramidate conjugated to the AChE active site. ${ }^{5}$ Furthermore, Musilek et al. ${ }^{6}$ reported that steric hindrance induced by tabun-inhibited AChE led to partial closing of the enzyme's active site, so that only few bispyridinum oximes could enter the active site to reactivate tabun-inhibited AChE. The most promising of the standard oximes, HI-6, cannot reactivate tabun-inhibited enzyme, though it exerts a significant protection via different mechanisms. ${ }^{7}$ Oxime TMB-

\footnotetext{
$\dagger$ Presented at the $10^{\text {th }}$ Congress of the Croatian Society of Biochemistry and Molecular Biology held in Opatija, Croatia, September 15-18, 2010.

* Author to whom correspondence should be addressed. (E-mail: suzana@imi.hr)
} 
4 effectively reactivates tabun-inhibited AChE, and it also reduces or alleviates the toxic effects of tabun, showing beneficial effects in the treatment of tabunpoisoned animals. ${ }^{8-9}$ But it is the most toxic oxime of the four mentioned oximes. ${ }^{10}$ Therefore, there is still a need to develop not only the most effective, but also the least toxic organophosphate antidote.

Recent in vitro and in vivo studies draw attention to oxime [(E)-1-(4-carbamoylpyridinium)-4-(4-hydroxyiminomethylpyridinium)-but-2-ene dibromide](K203) as a very potent reactivator of tabun-inhibited AChE. ${ }^{11-14}$ In this study, we used rats as the experimental model to broaden our knowledge about the antidotal potency of K203 as well as to evaluate the existence of oxidative stress and its involvement in OP toxicity. Namely, it is nowadays known that additional noncholinergic mechanisms contribute to OP toxicity. ${ }^{15}$ Some of these mechanisms include excessive generation of free radicals. ${ }^{16}$ If the free radical production overwhelms the endogenous antioxidant levels, free radical activity can lead to cell damage, known as oxidative stress. Thus, rat plasma samples were analyzed for total cholinesterase (ChE) activity and levels of oxidative stress markers (concentration of thiobarbituric reactive substances, TBARS as a measure of lipid peroxidation and activity of the antioxidant enzyme superoxide dismutase, SOD) after exposure to tabun, oxime, atropine or their combinations.

\section{EXPERIMENTAL}

\section{Chemicals}

The structure of the studied oxime K203 and tabun is given in Figure 1. K203 [(E)-1-(4-carbamoylpyridinium)-4-(4-hydroxyiminomethylpyridinium)-but-2-ene dibromide] was prepared as described earlier ${ }^{17}$ at the Faculty of Military Health Sciences, Hradec Králové, Czech Republic. It was kept at room temperature and was dissolved in distilled water or atropine immediately before use. Doses used in the experiments were 8.41 and $42.05 \mathrm{mg} \mathrm{kg}^{-1}$ body weight, corresponding to 5 and $25 \%$ of its $\mathrm{LD}_{50}\left(\mathrm{LD}_{50}=168.2 \mathrm{mg} \mathrm{kg}^{-1}\right)$, respectively. ${ }^{12}$ Atropine sulphate (Kemika, Zagreb, Croatia) was dissolved in distilled water $\left(5.0 \mathrm{mg} \mathrm{mL}^{-1}\right)$ and applied at a dose of $10 \mathrm{mg} \mathrm{kg}^{-1}$ body weight. Tabun [ethyl $N, N$ dimethylphosphoroamidocyanidate] was purchased from the NC Laboratory, Spiez, Switzerland. Stock solution of $5000 \mu \mathrm{g} \mathrm{mL}^{-1}$ tabun was prepared in isopropyl alcohol. Further dilutions were made in saline shortly before use. Rats were poisoned with a sublethal dose (75 \% LD $\mathrm{LD}_{50} ; \mathrm{LD}_{50}=317.5 \mu \mathrm{g} \mathrm{kg}^{-1}$ body weight) of tabun. Other chemicals and reagents used were purchased form Sigma Chemical Co., St. Louis, MO, USA, unless specified otherwise.
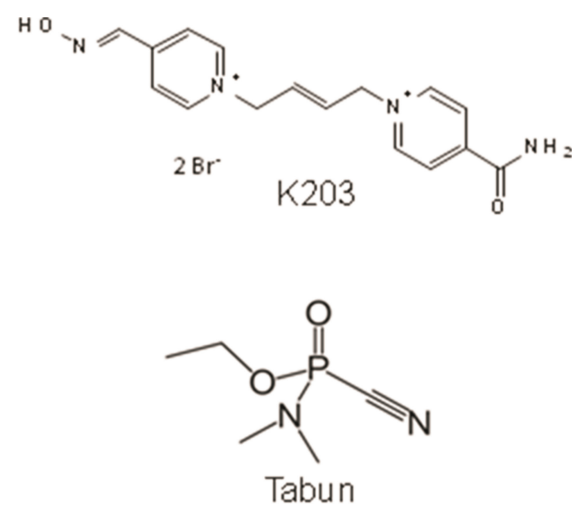

Figure 1. Structure of the tested oxime and tabun.

\section{Animals}

Adult male Wistar rats were obtained from the Institute for Medical Research and Occupational Health, Zagreb, Croatia, and were selected for weight (240-280 g). The animals were kept in Macrolone cages under controlled conditions (room temperature $21{ }^{\circ} \mathrm{C}$, light and dark cycles exchanging every $12 \mathrm{~h}$ ), received a standard diet for laboratory rodents (Sljeme, Zagreb, Croatia) and had free access to water. The rats $(\mathrm{N}=128)$ were randomly divided into groups of four animals each. This study was performed with the approval of the Ethical Committee of the Institute for Medical Research and Occupational Health, Zagreb, Croatia.

\section{Treatment}

For the purpose of biochemical experiments, rats received a subcutaneous (s.c.) sublethal dose $\left(75 \% \mathrm{LD}_{50}\right)$ of tabun. The therapeutic efficacy of oxime K203 was tested as follows: a) K203 alone ( 5 or $25 \% \mathrm{LD}_{50}$ ) one minute after tabun poisoning, b) K203 (5 or $25 \% \mathrm{LD}_{50}$ ) plus atropine one minute after tabun poisoning. Other three groups of animals were treated with oxime only ( 5 or $25 \% \mathrm{LD}_{50}$ ) or just atropine. Oxime and atropine were administered intraperitoneally (i.p.). Control rats received i.p. $2 \mathrm{~mL} \mathrm{~kg}^{-1}$ of saline (i.p.). All animals were humanely euthanized $0.5,1,6$ or $24 \mathrm{~h}$ after the treatment. Blood samples were obtained directly from the heart. Plasma was separated by centrifugation and stored at $-20{ }^{\circ} \mathrm{C}$ until further processing.

\section{Cholinesterase Activity}

The catalytic activity of cholinesterases in plasma was determined spectrophotometrically using the method of Ellman et al. ${ }^{18}$ which is based on the use of 5,5'dithiobis (2-nitrobenzoic acid) (DTNB) as a thiol reagent in the presence of acetylthiocholine iodide (ATCh) as substrate. The measured activity represented total cholinesterase activity in plasma and it was expressed as IU $\mathrm{g}_{\text {protein }}{ }^{-1}$. 


\section{Lipid Peroxidation}

Concentration of thiobarbituric reactive substances (TBARS), as a measure of lipid peroxidation, was determined using a modification of the method of Drury et $a l .{ }^{19}$ Concentration of TBARS was calculated using standard curves of increasing 1,1,3,3-tetramethoxypropane concentrations and was expressed as $\mu \mathrm{mol} \mathrm{L}{ }^{-1}$.

\section{Superoxide Dismutase Activity}

Plasma total SOD activity was measured spectrophotometrically according to Flohé and Ötting. ${ }^{20}$ The reduction rate of cytochrome $c$ by superoxide radicals was monitored at $550 \mathrm{~nm}$ utilizing the xantinexantine oxidase system as the source for $\mathrm{O}_{2}{ }^{--}$. SOD competed for superoxide and decreased the reduction rate of cytochrome $c$. One unit of SOD was defined as the amount of enzyme that inhibits the rate of cytochrome $c$ reduction by $50 \%$. Enzyme activity was expressed as IU grotein $^{-1}$.

\section{Protein Quantification}

Determination of protein concentration was carried out according to the method of Bradford ${ }^{21}$ using bovine serum albumin as standard.

\section{Statistical Analysis}

Data were expressed as median \& interquartile range. Statistical significance was determined using the MannWhitney test and differences were considered significant when $P<0.05$.

\section{RESULTS AND DISCUSSION}

Organophosphorus compounds poisoning is a major public health concern. Indeed, self-poisoning by OP pesticides is responsible for some 200,000 deaths a year in the world ${ }^{22}$ and some potent chemical warfare agents are today considered as potential terrorist agents. Although prevention and therapy of poisoning with OP compounds has been a subject of many studies, therapy with atropine and oximes still leaves much to be desired. Pyridinium oximes have probably been known for more than half a century, but there is no single compound that can counteract the toxic potential of each OP. This was the reason for synthesizing a new group of bispyridinium oximes as potential antidotes against OP poisoning. In the Czech Republic, considerable efforts have been invested into the development of new reactivators to be used in cases of tabun poisoning. The best recommended oxime of the third generation of the so called K-oximes for tabuninhibited AChE seems to be oxime K203. ${ }^{23}$ In our recently published study, we obtained a high percentage of reactivation of tabun-inhibited human erythrocyte AChE in a short time with micromolar concentrations of K203. ${ }^{12}$ Furthermore, K203 in combination with atropine given one minute after tabun poisoning ensured survival of all mice at an $8.0 \mathrm{LD}_{50}$ dose of tabun. $^{12}$ In the last three years, similar results were also published by other researchers. ${ }^{6,11,13,14}$

The primary objective of this study was to determine the ability of K203 to reactivate plasma cholinesterase in tabun-poisoned rats. We characterized poisoning through the intensity of toxic signs and through the level of ChE inhibition. Plasma samples were measured for total cholinesterase activities. Namely, rodent plasma contains higher amount of butyrylcholinesterase than acetylcholinesterase. ${ }^{24} \mathrm{OP}$ compounds inhibit both enzymes although sometimes at different rates. ${ }^{1}$ Administration of a single sublethal dose of tabun induced twitch potentiation, fasciculations and muscular weakness. Signs of toxicity reached maximum $15 \mathrm{~min}$ to $1 \mathrm{~h}$ after tabun administration. Changes in cholinesterase activity are shown in Figure 2. Tabun produced maximum $\mathrm{ChE}$ inhibition at $1 \mathrm{~h}$ after exposure, while after $24 \mathrm{~h}$ plasma activities in poisoned rats increased. Administration of atropine or oxime alone did not affect the ChE activity in rat plasma. What is even more important is that oxime K203 did not modify enzyme activity even at a dose of $25 \%$ of its $\mathrm{LD}_{50}$ although one could expect so because it is known that oximes are reversible inhibitors of ChE. ${ }^{4}$ As regards therapeutic efficacy, K203 either alone or in combination with atropine significantly increased ChE activity at 0.5 and $1 \mathrm{~h}$, compared to rats poisoned with tabun. There were no considerable differences in responses between the applied doses of K203. Judging by the followed time course of changes for ChE activity, the pharmacological effect of K203 alone seems to be almost equal to the effect of the K203 and atropine combination. Although the therapeutic effects of pyridinium oximes can be greatly improved if given with atropine, our results confirmed the hypothesis that oxime reactivation is the only treatment modality for OP exposure when recovery of the original condition of an active enzyme molecule is desired. Mechanistic studies of oxime action have shown that oximes as reversible ligands may bind either at the catalytic site or at the allosteric site, or at both sites of the enzyme, thus explaining the mechanism of reactivation or protection afforded by oxime administration. ${ }^{25}$ Taking in account the underlying mechanisms, it becomes clear that stereochemical arrangements of oximes play an important role in their therapeutic efficacy. The structure of oxime K203 containing the functional oxime group at position four at one pyridinium ring and carbamoyl group at the 
$30 \mathrm{~min}$

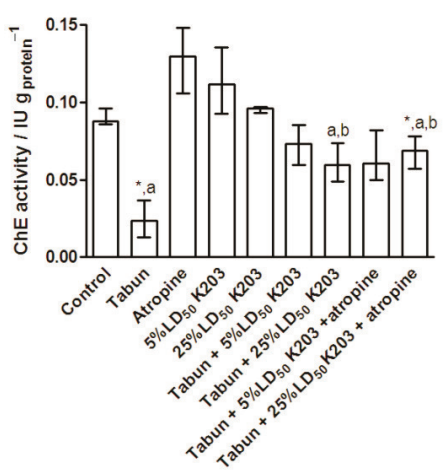

6h

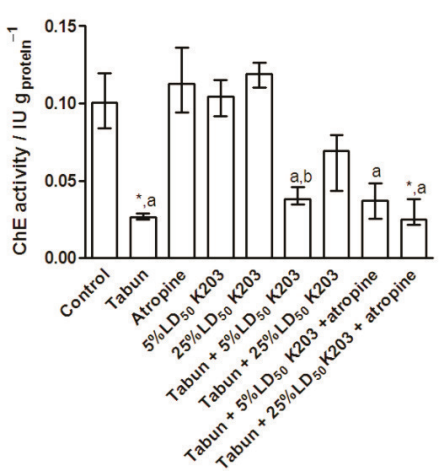

1h

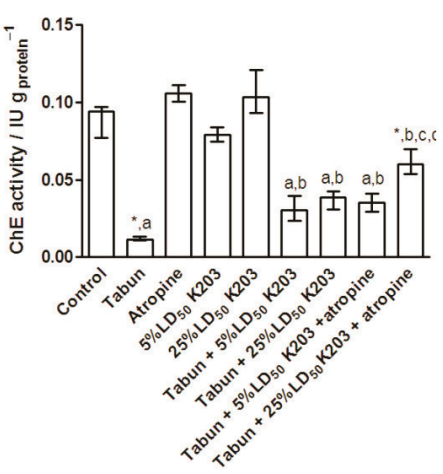

$24 \mathrm{~h}$

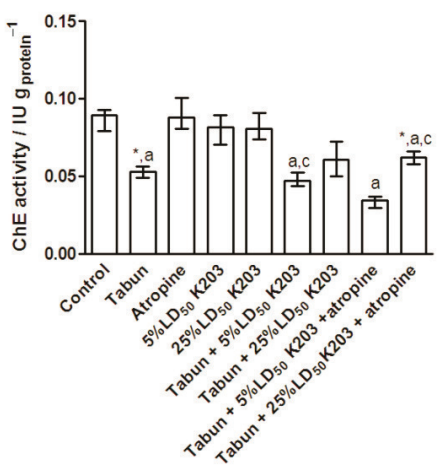

Figure 2. Changes in cholinesterase activity in plasma of rats following the treatment with tabun $\left(75 \% \mathrm{LD}_{50}\right)$, oxime $\mathrm{K} 203(5$ or $\left.25 \% \mathrm{LD}_{50}\right)$, atropine $\left(10 \mathrm{mg} \mathrm{kg}^{-1}\right)$ and their combinations. Significantly increased values $(P<0.05)$ are: (a) compared to the control; (b) compared to tabun-poisoned rats; (c) compared to rats given $5 \% \mathrm{LD}_{50} \mathrm{~K} 203$ plus atropine as therapy; (d) compared to rats given $25 \% \mathrm{LD}_{50} \mathrm{~K} 203$ as therapy. Asterisk denotes data from Ref. 12.

other pyridinium ring with $(E)$-but-2-ene linker suggests K203 as a potential candidate for treatment of tabun intoxication. According to QSAR studies with a set of 11 oximes, Bhattacharjee et al. ${ }^{26}$ reported that in K203 the distance between the bisquaternary nitrogen atoms $\left(\mathrm{N}^{+}-\mathrm{N}^{+}\right)$, increased hydrophilicity, lower surface area, and lower nucleophilic power of the oxime oxygen together provide the necessary stereoelectronic attributes that enable K203 to be a better and less toxic reactivator for tabun-inhibited AChE. According to our previously published results, K203 showed moderate toxicity in mice and rats. ${ }^{12}$ Since most OP nerve agents are volatile and hydrophobic, they cross the blood-brain barrier and become sequestered in the brain and periphery, from which they slowly relieve. Hence the OP inactivation in plasma must be efficient. At this point, we demonstrated that administration of oxime K203 one minute after tabun poisoning seems to be effective in restoring the activity of cholinesterases in rat plasma. Even more, when applied at a dose $5 \%$ of its $\mathrm{LD}_{50}$, which is considered to be a medical dose, ${ }^{27}$ this oxime was also capable to display excellent levels of reactivation.
Since other mechanisms, notably oxidative stress, have been proposed for the toxicity of OP in animals and humans, another objective of this study was to estimate the levels of oxidative stress markers. The lipophilic nature of OP facilitates their interaction with the cell membrane and leads to perturbations of the phospholipid bilayer structure. ${ }^{28}$ It was therefore decided to determine the concentration of thiobarbituric reactive substances (TBARS) as a measure of lipid peroxidation (Figure 3), and to see whether there is any difference in this parameter upon tabun poisoning and therapies administrated to rats. Single application of compounds, even exposure to tabun, did not increase plasma TBARS concentrations when compared to control. On the other hand, the same range of values or higher than control was obtained in plasma samples of rats that received therapy one minute after tabun poisoning. In most cases in plasma of animals that received therapy TBARS concentration was significantly higher than the TBARS concentration in plasma of tabun poisoned rats. In animals sacrified $30 \mathrm{~min}$ after the treatment there were no differences between therapy containing K203 alone and therapy combined of K203 and atropine. Also, in that 
$30 \mathrm{~min}$

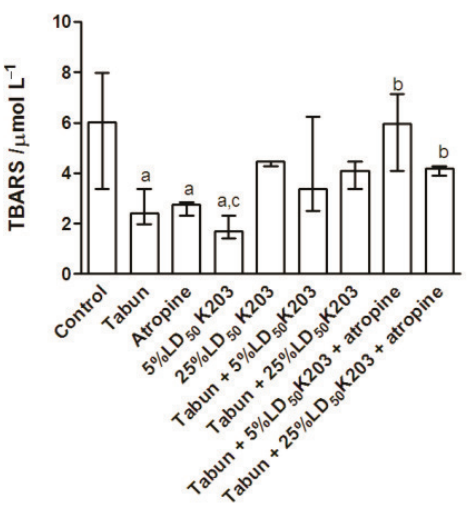

$6 \mathrm{~h}$

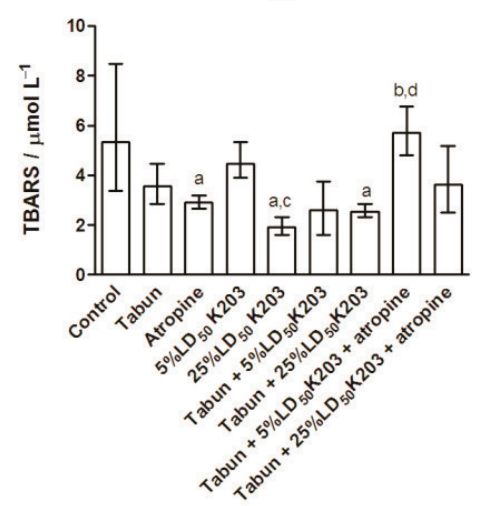

$1 \mathrm{~h}$

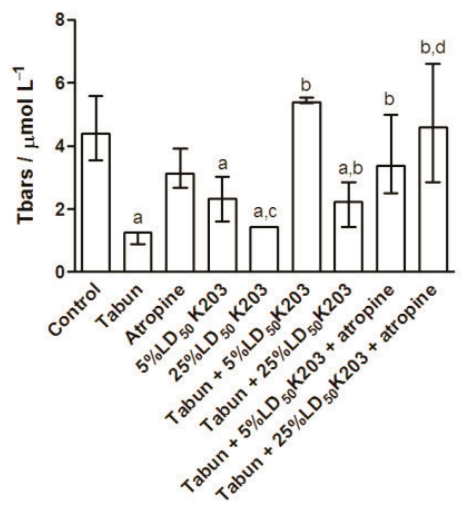

$24 h$

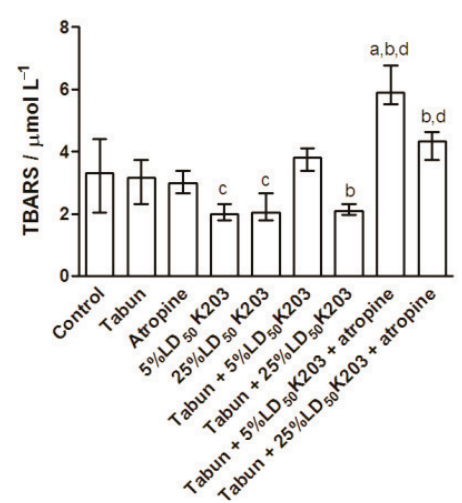

Figure 3. Changes in the TBARS concentration in plasma of rats following the treatment with tabun $\left(75 \% \mathrm{LD}_{50}\right)$, oxime $\mathrm{K} 203(5$ or $\left.25 \% \mathrm{LD}_{50}\right)$, atropine $\left(10 \mathrm{mg} \mathrm{kg}^{-1}\right)$ and their combinations. Significantly different values $(P<0.05)$ are: (a) compared to the control; (b) compared to tabun-poisoned rats; (c) compared to rats administrated atropine alone; (d) compared to rats given K203 alone as therapy.

time period no significant dose dependence considering oxime K203 was observed. During longer time periods difference in TBARS concentration depending on the dose of oxime was noticed, and generally the highest increase of TBARS concentration was observed in plasma of rats that received therapy combined of oxime and atropine immediately after tabun poisoning. Unfortunately, without a detailed insight into metabolizing processes we cannot claim that there is a negative synergism of tabun, oxime and atropine combination that could cause elevation of free radicals and consenquently lipid peroxidation. To the best of our knowledge, there are no published data on the relationship of this parameter with atropine and oxime treatment in the acute OP poisoning. There is however a study reported by Abu-Qare and Abou-Donia ${ }^{29}$ that demonstrated potential of prophylactic drug pyridostigmine bromide and nerve agent sarin to increase the urinary biomarkers of oxidative stress after combined exposure in rats. Nevertheless, our study has demonstrated that acute exposure to sublethal dose of tabun did not increase concentration of lipid peroxidation products. Although many studies have revealed histopatological and biochemical changes in target tissues through OP induced oxidative stress, ${ }^{30}$ there are a few reports showing no oxidative stress upon exposure to OP in in vivo models. For example, Hazarika et $a l .{ }^{31}$ found that anilofos cannot cause lipid peroxidation in cell membranes of liver and brain in exposed rats. Silva et al. $^{32}$ demonstrated that no change is observed in the biochemical parameters related to oxidative stress in malathion exposed mice. Important fact for discussion is that much of the data regarding involvement of oxidative stress in OP toxicity were gathered from the studies with OP pesticides. ${ }^{30}$ Since several phenomena appear to differentiate nerve agent exposure from exposure to pesticides, extrapolation to predictions of effects from nerve agents may be subject to risk. The sparse available data suggest that large toxic doses of OP nerve agents cause acute necrotic neuronal cell death, whereas sublethal doses produce apoptotic neuronal cell death and involve oxidative stress. ${ }^{33}$ 
$30 \mathrm{~min}$

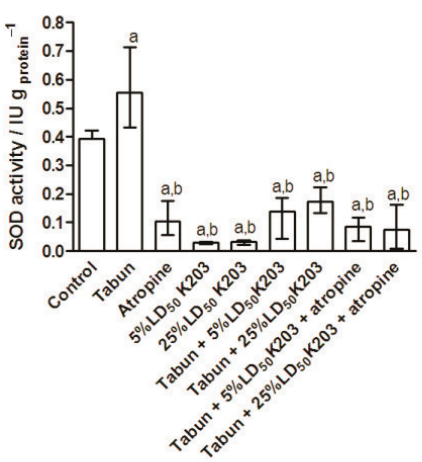

$6 \mathrm{~h}$

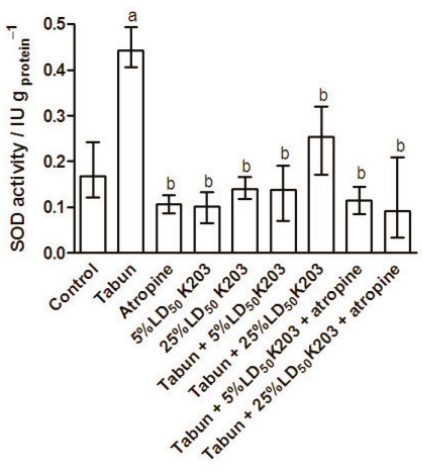

$1 \mathrm{~h}$

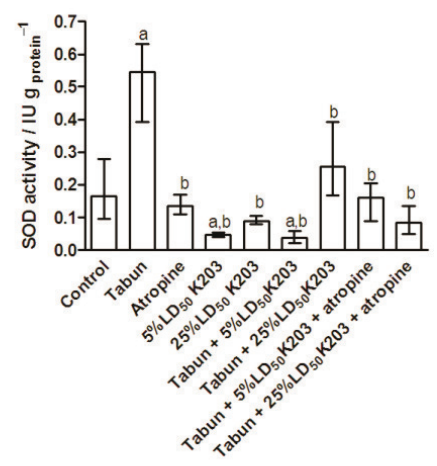

$24 \mathrm{~h}$

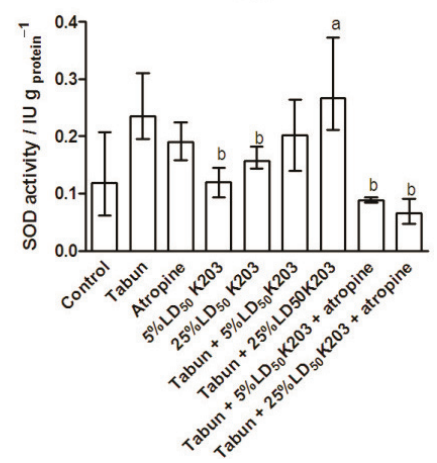

Figure 4. Changes in superoxide dismutase (SOD) activity in plasma of rats following the treatment with tabun $\left(75 \% \mathrm{LD}_{50}\right)$, oxime K203 (5 or $\left.25 \% \mathrm{LD}_{50}\right)$, atropine $\left(10 \mathrm{mg} \mathrm{kg}^{-1}\right)$ and their combinations. Significantly increased values $(P<0.05)$ are: (a) compared to the control; (b) compared to tabun-poisoned rats.

In view of the possible oxidative stress involved in OP poisoning, rat plasma samples were also analyzed for the activity of the antioxidant enzyme superoxide dismutase. SOD is considered to be one of the first lines of defence against deleterious effects of oxyradicals in the cell by catalyzing the dismutation of superoxide radicals. ${ }^{31}$ As seen from Figure 4, SOD activities in plasma samples of control rats progressively decreased up to $24 \mathrm{~h}$. The same trend was noticed in plasma of tabun poisoned rats but during all time periods tabun poisoning resulted with significantly higher SOD activities than control. Interestingly, administration of atropine or oxime ( 5 or $25 \% \mathrm{LD}_{50}$ ) alone caused an opposite response of plasma SOD activity. During the first hour SOD activities in rats that received either atropine or oxime were significantly lower than in controls. An increase was noticed at 6 and $24 \mathrm{~h}$ following atropine or oxime administration and almost the same values of SOD activities as in the control groups were achieved. The same response was observed in rats that received therapies applied one minute after tabun poisoning. Yet it is important to emphasize that high SOD activities detected in plasma samples of rats poisoned with a sublethal dose of tabun significantly differ from all other treatments. Thus, we could say that tabun poisoning induced generation of free radicals and led to a marked increase in enzymatic antioxidant activity. It seems that this early response of SOD has an impact on other processes related to oxidative stress, i.e. by neutralizing superoxide radicals it prevents lipid peroxidation. Accumulation of lipid peroxidation products is believed to be a major contributor to the loss of cell function under oxidative stress conditions. ${ }^{30}$ An increase in enzymatic and nonenzymatic antioxidants of defence mechanisms might be an indicator of the decrease in TBARS level. According to our results it is possible that the induction of SOD activity prevented lipid peroxidation after tabun poisoning. However, the studied effect of acute exposure to a sublethal dose of tabun on oxidative stress induction is probably not the best approach, presumably because a $24 \mathrm{~h}$ period and a single dose of tabun are inadequate conditions to delineate the role of oxidative stress in the toxicity of OP.

\section{CONCLUSIONS}

Together with published data on K203 efficacy, our results indicate the high potency of this oxime in coun- 
teracting tabun poisoning. Judging by the followed time course of changes for $\mathrm{ChE}$ activity in rat plasma, it seems that the therapeutic effect of this oxime is indeed related to the reactivation of tabun-phoshorylated enzyme.

Based on simultaneous measurements of TBARS and SOD, we can conclude that tabun poisoning, but also application of therapy (oxime alone or oxime plus atropine) immediately after tabun poisoning, could generate free radical species that may cause oxidative stress in rats. Finally we suggest that the in vivo effects of these componds should be investigated further to better understand underlying mechanisms that could lead to development of better antidotes.

Acknowledgements. The authors thank Jasna Mileković, Marija Kramarić and Mirjana Matašin for technical assistance. This work was supported by the Ministry of Science, Education and Sports of the Republic of Croatia (Grant No. 0220222148-2139) and Czech Grant Agency (Project No. 305/07/P162).

\section{REFERENCES}

1. T. C. Marrs, Pharmacol. Ther. 58 (1993) 51-66.

2. J. L. Sussman, M. Harel, F. Frolow, C. Oefner, A. Goldman, L. Toker, and I. Silman, Science 253 (1991) 872-879.

3. S. M. Somani and K. Husain, Low-level Nerve Agent Toxicity under Normal and Stressful Conditions, in: S.M. Somani and J.A. Jr. Romano (Eds.), Chemical Warfare Agents: Toxicity at Low Levels, CRS Press LLC, Boca Raton, Florida, 2001, pp. 83 -120.

4. H. Thiermann, L. Szinicz, F. Eyer, F. Worek, P. Eyer, N. Felgenhauer, and T. Zilker, Toxicol. Lett. 107 (1999) 233-239.

5. M. Eto, Organic and Biological Chemistry, in: G. Zweig (Ed.), The Organophosphorous Pesticides, CRC Press Inc. Cleveland, 1976 , p. 142.

6. K. Musilek, K. Kuča, D. Jun, and M. Dolezal, Curr. Org. Chem. 11 (2007) 229-238.

7. M. G. Hamilton and P. M. Lundy, Arch. Toxicol. 63 (1989) 144-149.

8. M. Maksimović, B. Bošković, L. Radović, V. Tadić, V. Deljac, and Z. Binenfeld, Acta Pharm. Jugoslav. 30 (1980) 151-160.

9. M. Jokanović, M. Maksimović, V. Kilibarda, D. Jovanović, and D. Savić, Toxicol. Lett. 85 (1996) 35-39.

10. M. P. Stojiljković and M. Jokanović, Arh. Hig. Rada Toksikol.
57 (2006) 435-443.

11. J. Kassa, J. Karasova, K. Musilek, and K. Kuča, Toxicol. 243 (2008) 311-316.

12. Z. Kovarik, A. Lucić Vrdoljak, S. Berend, M. Katalinić, K. Kuča, K. Musilek, and B. Radić, Toxicology 60 (2009) 19-25.

13. J. Kassa, J. Karasova, L. Vasina, J. Bajgar, K. Kuča, and K. Musilek, Drug Chem. Toxicol. 32 (2009) 128-138.

14. J. Kassa, J. Karasova, F. Caisberger, K. Musilek, K. Kuča, and Y. S. Jung, J. Enzyme Inhib. Med. Chem. 25 (2010) 480-484.

15. B. Antonijević and M. P. Stojiljković, Clin. Med. Res. 5 (2007) 71-82.

16. D. Milatović, R. C. Gupta, and M. Aschner, ScientificWorldJournal 6 (2006) 295-310.

17. K. Musilek, D. Jun, J. Cabal, J. Kassa, F. Gunn-Moore, and K. Kuča, J. Med. Chem. 50 (2007) 5514-5518.

18. G. L. Ellman, K. D. Courtney, V. J. Andres, and R. M. Featherstone, Biochem. Pharmacol. 7 (1961) 88-95.

19. J. A. Drury, J. A. Nycyk, and R. W. I. Cooke, Clin. Chim. Acta 263 (1997) 177-185.

20. L. Flohé and G. Ötting, Methods Enzymol. 105 (1971) 93-104.

21. M. M. Bradford, Anal. Biochem. 72 (1976) 248-254.

22. M. Eddleston, N. A. Buckley, P. Eyer and A. H. Dawson, Lancet 371 (2008) 597-607.

23. K. Kuča, K. Musilek, D. Jun, J. Bajgar, and J. Kassa, Novel oximes, in: R.C. Gupta (Eds.), Handbook of Toxicology of Chemical Warfare Agents, Academic Press-Elsevier, Amsterdam, 2009, pp. 997-1021.

24. B. Li, M. Sedlacek, I. Manoharan, R. Boopathy, E. G. Duysen, P. Masson, and O. Lockridge, Biochem. Pharmacol. 70 (2005) 1673-1684.

25. E. Reiner, Croat. Chem. Acta 59 (1986) 925-931.

26. A. K. Bhattacharjee, K. Kuča, K. Musilek, and R. K. Gordon, Chem. Res. Toxicol. 23 (2010) 26-36.

27. M. Pohanka, J. Zdarova Karasova, K. Musilek, K Kuca, and J. Kassa, J. Appl. Toxicol. 6 (2009) 483-488.

28. R. A. Videira, M. C. Antunes-Madeira, V. I. C. F. Lopes, and V. M. C. Madeira, Biochim. Biophys. Acta 1411 (2001) 360-368.

29. A. W. Abu-Qare and M. B. Abou-Donia, Toxicol. Lett. 123 (2001) 51-58.

30. K. Soltaninejad and M. Abdollahi, Med. Sci. Monit. 15 (2009) 75-90.

31. A. Hazarika, S. N. Sarkar, and M. Kataria, Indian J. Exp. Biol. 39 (2001) 1113-1117.

32. A. P. Silva, F. C. Meotti, A. R. S. Santos, and M. Farina, Neurotoxicol. 27 (2006) 1101-1105.

33. M. B. abou-Donia, Arch. Environ. Health 58 (2003) 484-497.

34. M. Romeu, M. Mulero, M. Giralt, J. Folch, M. R. Nogues, A. Torres, A. Fortuno, F. X. Sureda, M. Cabre, J. L. Paternain, and J. Mallol, Life Sci. 71 (2002) 1739-1749. 